\title{
Quantization of the scalar field in a static quantum metric
}

\author{
Z. Haba \\ Institute of Theoretical Physics, University of Wroclaw, \\ 50-204 Wroclaw, Plac Maxa Borna 9, Poland \\ e-mail:zhab@ift.uni.wroc.pl
}

\begin{abstract}
We investigate Hamiltonian formulation of quantum scalar fields in a static quantum metric. We derive a functional integral formula for the propagator. We show that the quantum metric substantially changes the behaviour of the scalar propagator and the effective Yukawa potential.
\end{abstract}

\section{Introduction}

There is an old idea coming from Landau, Pauli and others ( see the review of the problem in 1957 by Deser [1]) that quantization of gravity could cure the ultraviolet divergencies in quantum field theory of matter fields interacting with gravity. Since then this inspiring idea has been followed by other authors (let us mention [2]-[3]). However, in general we find their approximate methods unreliable. In our earlier papers [4]-[6] we have investigated an interaction of a scalar field with a quantum or random metric in a functional integral formulation using the proper time representation for the propagator. The interaction of gravity with the scalar field has been treated non-perturbatively. We explicitly calculated an average over the metric field. For the reason of a mathematical consistency the functional measure of the metric field did not satisfy conditions required for the quantum field. In such a case it remained unclear whether the results could have been derived in a unitary framework of Hamiltonian dynamics. In this paper we study a similar model in the Hamiltonian formulation and relate the results to the ones of the functional integral form. We make an assumption that the metric does not depend on some coordinates in order to be able to perform explicit calculations. In particular, this assumption can be realized by a choice of a static metric. Then, we discuss in detail the model of a scalar field interacting with a static quantized metric. We are interested in a model where the quantum metric comes from a time-zero quantum field. In 
general, such a field evolves with time. In order to simplify the model we neglect this time evolution. A perturbation of the free Hamiltonian by a quantized metric is a singular perturbation problem. So, we first start with a regularized metric which is an analytic function. We discuss the operator Hamiltonian formalism in the Fock space. We express the expectation values of the time-ordered products of the scalar field in the Fock space at some special complex points by means of a rigorous version of the Feynman integral. Then, the average over the metric field is calculated. Finally, the regularization of the metric field can be removed. We discuss in some detail an example of the metric being a threedimensional free quantum field. We show that an interaction with such a quantum metric substantially changes the short distance behaviour of the scalar propagator. The Hamiltonian approach allows to calculate the S-matrix and to calculate the effective two-body potential. We discuss these problems briefly.

\section{The Hamiltonian approach}

We consider the usual Lagrangian for the scalar field

$$
L=g^{A B} \partial_{A} \phi \partial_{B} \phi
$$

We assume that the metric $g$ in $D$ dimensions (with the decomposition $D=$ $d+(D-d)$ of the coordinates $x=(\mathbf{x}, X))$ depends only on $d$ spatial coordinates $\mathbf{x}$ and is of the block diagonal form $\left(g^{A B}\right)=\left(g^{j k}(\mathbf{x}), g^{\mu \nu}(\mathbf{x})\right)$ where $g^{A B}=$ $\eta^{A B}+h^{A B}$ and $\eta^{D D}=-1$ with the time $t=X_{D}$ (we shall concentrate here on the case $D=4$ and $d=3)$. Let $f_{\alpha} \exp \left(-i \omega_{\alpha} t\right)$ be the set of positive frequency solutions (it is clear what it means because the metric is time-independent) of the wave equation

$$
-2 \mathcal{A} \phi \equiv \partial_{A}\left(g^{A B} \sqrt{g} \partial_{B}\right) \phi=0
$$

where $g=-\operatorname{det}\left(g_{A B}\right)$.

We choose the normalization

$$
\int d \mathbf{x} \bar{f}_{\alpha} f_{\beta}=\frac{1}{\omega_{\alpha}} \delta_{\alpha \beta}
$$

We expand the solution $\phi$ into a complete set of positive $f_{\alpha} \exp \left(-i \omega_{\alpha} t\right)$ and negative energy solutions $\bar{f}_{\alpha} \exp \left(i \omega_{\alpha} t\right)$

$$
\phi(t, \mathbf{x})=\sum_{\alpha} f_{\alpha}(\mathbf{x}) a_{\alpha} \exp \left(-i \omega_{\alpha} t\right)+\bar{f}_{\alpha}(\mathbf{x}) a_{\alpha}^{+} \exp \left(i \omega_{\alpha} t\right)
$$

where $\left(a_{\alpha}, a_{\alpha}^{+}\right)$are the usual annihilation and creation operators in a Fock space $\mathcal{F}_{s c}$

$$
\left[a_{\alpha}, a_{\beta}^{+}\right]=\delta_{\alpha \beta}
$$


The Hamiltonian describing the time evolution (3) reads

$$
H=\sum_{\alpha} \omega_{\alpha} a_{\alpha}^{+} a_{\alpha}
$$

Let $|0\rangle_{s}$ be the Fock vacuum for the scalar field. We can compute the timeordered product

$$
\left\langle 0\left|T\left(\phi(x) \phi\left(x^{\prime}\right)\right)\right| 0\right\rangle_{s}=\sum_{\alpha}\left(f_{\alpha}(\mathbf{x}) \bar{f}_{\alpha}\left(\mathbf{x}^{\prime}\right)+f_{\alpha}\left(\mathbf{x}^{\prime}\right) \bar{f}_{\alpha}(\mathbf{x})\right) \exp \left(-i \omega_{\alpha}\left|t-t^{\prime}\right|\right) \equiv G_{F}\left(x, x^{\prime}\right)
$$

$G_{F}$ is the solution of the equation

$$
\partial_{A}\left(g^{A B} \sqrt{g} \partial_{B}\right) G_{F}=-i \delta
$$

It follows that $\left\langle 0\left|T\left(\phi(x) \phi\left(x^{\prime}\right)\right)\right| 0\right\rangle_{s}=-i \square_{g}^{-1}\left(x, x^{\prime}\right)$, where $\square_{g}$ denotes the wave operator on a manifold. As usual for the fields linear in creation and annihilation operators the higher order correlation functions are expressed by the two-point function. Then, the subsequent average over the metric concerns an average of the propagators $G_{F}$.

We can also derive the time evolution (3) from the canonical formalism

$$
[\phi(\mathbf{x}), \Pi(\mathbf{y})]=i \delta(\mathbf{x}-\mathbf{y})
$$

and

$$
H=\int d \mathbf{x} \sqrt{g}\left(\frac{-g_{00}}{g}(\mathbf{x}) \Pi(\mathbf{x}) \Pi(\mathbf{x})+g^{j k}(\mathbf{x}) \partial_{j} \phi(\mathbf{x}) \partial_{k} \phi(\mathbf{x})\right)
$$

It is understood that there is a normal ordering of the bilinear forms in $\Pi$ and $\phi$ in $H$. The normal ordering requires metric-dependent counterterms. Such terms would change the gravitational dynamics but in our approximation we have to neglect altogether the gravitational dynamics later on in order to perform explicit calculations. The non-linear local function of the quantum metric field in $H$ itself needs a proper definition. The scalar field Hamiltonian leads to the wave equation (2) no matter what this definition is ( after the normal ordering of the scalar fields ).

If $g^{A B}=\eta^{A B}+h^{A B}$ then $H=H_{0}+H_{I}$ where $H_{I}$ is the interaction Hamiltonian for the computation of the $S$-matrix (see, eq.(8) below).

The metric $h^{\mu \nu}$ may be operator-valued but we assume that the operators $h^{\mu \nu}$ commute ( $h^{j k}$ are treated as a fixed classical background). In particular, we consider a model of relativistic fields $h^{\mu \nu}(\mathbf{x}, t)$ such that $h^{\mu \nu}(\mathbf{x})$ are timezero fields which live in the Fock space $\mathcal{F}_{g r}$ and are expanded in creation and annihilation operators ( we have in mind a model of a canonical free field)

$$
h^{\mu \nu}(t, \mathbf{x})=\sum\left(f_{\alpha}^{\mu \nu}(\mathbf{x}) \exp \left(-i \nu_{\alpha} t\right) c_{\alpha}+\exp \left(i \nu_{\alpha} t\right) \bar{f}_{\alpha}^{\mu \nu}(\mathbf{x}) c_{\alpha}^{+}\right)
$$


where $f_{\alpha}^{\mu \nu}(\mathbf{x}) \exp \left(-i \nu_{\alpha} t\right)$ are solutions of the wave equation on a certain classical background. The Hamiltonian for the metric field is

$$
H_{g}=\sum_{\alpha} \nu_{\alpha} c_{\alpha}^{+} c_{\alpha}
$$

If the metric $h$ is defined on $R^{d}$ (a flat background metric) then we have $\nu_{\alpha}=c|\mathbf{k}|$ and $\alpha=(\sigma, \mathbf{k})$ where $\sigma$ is a polarization index, $\mathbf{k}$ is the momentum and

$$
f_{\alpha}^{\mu \nu}=P_{\sigma}^{\mu \nu}(\mathbf{k})(2 \pi)^{-\frac{d}{2}}(c|\mathbf{k}|)^{-\frac{1}{2}} \cos (\mathbf{k x})
$$

where $P$ depends on the choice of coordinates (the gauge)[7]. We consider quantum gravitons at the temperature $\mathrm{T}$ described by the Gibbs density matrix $\exp \left(-\beta H_{g}\right)$ where $\frac{1}{\beta}=k_{B} T$ and $k_{B}$ is the Boltzman constant. Then, the time ordered product of the metric fields reads

$$
\begin{aligned}
& \mathcal{D}^{\mu \nu ; \gamma \rho}\left(x-x^{\prime}\right) \equiv\left\langle 0\left|T\left(\exp \left(-\beta H_{g}\right) h^{\mu \nu}(x) h^{\gamma \rho}\left(x^{\prime}\right)\right)\right| 0\right\rangle_{g}= \\
& (2 \pi)^{-d} \hbar \int d \mathbf{k}(c|\mathbf{k}|)^{-1} \mathcal{P}^{\mu \nu ; \gamma \rho}(\mathbf{k}) \cos \left(\mathbf{k}\left(\mathbf{x}-\mathbf{x}^{\prime}\right)\right) \exp \left(-i c|\mathbf{k}|\left|t-t^{\prime}\right|\right)+ \\
& (2 \pi)^{-d} \hbar \int d \mathbf{k}(c|\mathbf{k}|)^{-1} \mathcal{P}^{\mu \nu ; \gamma \rho}(\mathbf{k}) \cos \left(\mathbf{k}\left(\mathbf{x}-\mathbf{x}^{\prime}\right)\right)(\exp (c \hbar \beta|\mathbf{k}|)-1)^{-1} \cos \left(c|\mathbf{k}|\left(t-t^{\prime}\right)\right)
\end{aligned}
$$

where

$$
\mathcal{P}^{\mu \nu ; \gamma \rho}=\sum_{\sigma} P_{\sigma}^{\mu \nu} P_{\sigma}^{\gamma \rho}
$$

depends on the gauge chosen for the metric field. In the limit $\hbar \rightarrow 0$ (or $T \rightarrow \infty$ ) we obtain the classical correlations resulting from the classical Gibbs distribution $\exp \left(-\beta \int \nabla h \nabla h\right)$ of the initial values for the gravitational field. Hence, the covariance behaves as

$$
|| \mathbf{x}-\mathbf{x}^{\prime}\left|-c\left(t-t^{\prime}\right)\right|^{-d+2}
$$

With a general time-independent background metric we should replace $c|\mathbf{k}|$ by $\nu_{\alpha}$ and $\mathcal{P} \cos \left(\mathbf{k}\left(\mathbf{x}-\mathbf{x}^{\prime}\right)\right)$ by

$$
\sum_{\alpha}\left(f_{\alpha}^{\mu \nu}(\mathbf{x}) \bar{f}_{\alpha}^{\gamma \rho}\left(\mathbf{x}^{\prime}\right)+f_{\alpha}^{\gamma \rho}\left(\mathbf{x}^{\prime}\right) \bar{f}_{\alpha}^{\mu \nu}(\mathbf{x})\right)
$$

in the formula for the temperature Green's functions . The short distance behaviour remains the same.

Instead of the thermodynamic equilibrium described by the Gibbs distribution we may consider a process of dissipation corresponding to an absorption of gravitational waves. Then, an absorption and emission of gravitational waves may lead to a time-independent (equilibrium) distribution of waves at large time, e.g., if the dissipation of gravitational energy is of the diffusive type, then for long times we could obtain

$$
\frac{d g}{d t}=\Gamma \triangle g+\Theta
$$


where the $\Gamma$-term describes a dissipation. If in the momentum (equiv.position) space the correlations of $\Theta$ have a $\delta$-type distribution then the invariant measure for the distribution of waves is the same as the one for the time-zero field at high temperature,i.e., $\triangle^{-1}(\mathbf{x}, \mathbf{y}) \simeq|\mathbf{x}-\mathbf{y}|^{-d+2}$. In any case in sec. 4 we assume the $|\mathbf{x}-\mathbf{y}|^{-d+2}$ behaviour of the metric correlations no matter where it comes from.

The $S$-matrix can be calculated from its generating functional in the interaction picture

$$
\begin{aligned}
& \left\langle 0\left|T\left(\exp \left(-i \int\left(H_{I}(s)+\int d \mathbf{x} J(s, \mathbf{x}) \phi(s, \mathbf{x})\right) d s\right)\right)\right| 0\right\rangle \\
& =\left\langle 0\left|\operatorname{det} \mathcal{A}^{-\frac{1}{2}} \exp \left(-\frac{1}{2} J G_{F} J\right)\right| 0\right\rangle_{g}
\end{aligned}
$$

In this formula $\Pi$ and $\phi$ on the l.h.s. undergo the free Hamiltonian evolution but (as a simplification of our model) $g$ does not change in time. In sec.4 we calculate the expectation values of $G_{F}$ under the assumption that $g$ is Gaussian $d=3$ and the propagator of $g$ is $|\mathbf{x}-\mathbf{y}|^{-1}$. From eq.(8) it can be seen that even if $g$ is the free field then the expectation values over $g$ of the Green's functions do not reduce to Gaussian integrals because of the $g$-dependence of $\operatorname{det} \mathcal{A}$. In order to calculate an average over $g$ we can apply the formula

$$
\operatorname{det} \mathcal{A}^{-\frac{1}{2}}=\exp \left(-\frac{1}{2} \operatorname{Tr} \ln \mathcal{A}\right)=\exp \left(\frac{1}{2} \operatorname{Tr} \int d m^{2}\left(\mathcal{A}+m^{2}\right)^{-1}\right)
$$

subsequently expanding the exponential. Such an expansion corresponds to an expansion in the number of closed scalar loops.

\section{The Feynman integral for the propagator}

We apply a representation of the Feynman integral by means of stochastic processes [8]-[9]. For a general metric we would need a Brownian motion on a manifold for this purpose [10]. In order to simplify the argument we consider the wave operator on a manifold with a particular metric in $D$ dimensions which in $D=d+1=4$ can be related to the one called conformally static metric in refs.[11]-[12]. We assume $g^{j k} \sqrt{g}=\delta^{j k}$ and $g_{00}=g^{j j}$ then

$$
\begin{aligned}
& -\mathcal{A}=\frac{1}{2} \partial_{A}\left(g^{A B} \sqrt{g} \partial_{B}\right) \\
& \equiv \frac{1}{2} \square_{D}+\frac{1}{2} \sum \hat{h}^{\mu \nu}(\mathbf{x}) \partial_{\mu} \partial_{\nu} \equiv \frac{1}{2} \hat{g}^{00} \partial_{0}^{2}+\frac{1}{2} \triangle
\end{aligned}
$$

where $\hat{g}^{A B}=\sqrt{g} g^{A B}$ and $\hat{h}^{A B}=\sqrt{g} h^{A B}$, and we split the $D$-coordinates $x=(\mathbf{x}, X)$ into $d$ coordinates $\mathbf{x}$ and $D-d$ coordinates $X$. Eq.(2) in $d=3$ can also be expressed in the form of a wave equation on a static threedimensional manifold (here $\triangle$ denotes the threedimensional Euclidean Laplacian)

$$
\left(\partial_{0}^{2}+\hat{g}_{00} \triangle\right) \phi \equiv\left(\partial_{0}^{2}+\hat{g}_{00} \partial_{k}\left(g^{k l} \sqrt{g} \partial_{l}\right)\right) \phi=0
$$


As an auxiliary tool for a calculation of the scalar propagator we consider the Schrödinger-type equation for the Hamiltonian $\mathcal{A}$

$$
i \partial_{\tau} \psi_{\tau}(\mathbf{x}, X)=\mathcal{A} \psi_{\tau}(\mathbf{x}, X)
$$

We can take the Fourier transform in $X$ because $g$ depends only on spatial coordinates $\mathbf{x}$. Then, eq.(9) takes the form

$$
i \partial_{\tau} \tilde{\psi}(\mathbf{x}, P)=\tilde{\mathcal{A}} \tilde{\psi}(\mathbf{x}, P)
$$

where

$$
\tilde{\mathcal{A}}=-\frac{1}{2} \triangle+\frac{1}{2} P_{\mu} P_{\nu} \hat{g}^{\mu \nu}(\mathbf{x})
$$

We consider the metrics $\hat{h}$ (as well as the initial states) which are analytic functions

$$
\hat{h}(\mathbf{x})=\int d \mathbf{p} \tilde{h}(\mathbf{p}) \exp (i \mathbf{p x})
$$

with the growth less than $\exp \left(\epsilon|\mathbf{z}|^{2}\right)$ (with arbitrarily small $\epsilon$ ) for a complex $\mathbf{z}$. Then, we can express the solution of eq.(9) by means of the Feynman integral

$$
\psi_{\tau}(\mathbf{x}, X)=\int d P \exp (i P X) E\left[M_{\tau} \tilde{\psi}(\mathbf{x}+\lambda \mathbf{b}(\tau), P)\right]
$$

where

$$
\lambda=\sqrt{i} \equiv \frac{1}{\sqrt{2}}(1+i)
$$

and

$$
M_{\tau}=\exp \left(-\frac{i}{2} P_{\mu} P_{\nu} \int_{0}^{\tau} \hat{g}^{\mu \nu}(\mathbf{x}+\lambda \mathbf{b}(s)) d s\right)
$$

Eq.(14) is understood as a limit $R \rightarrow \infty$ of a regularized expression with

$$
\hat{g}_{R}(\mathbf{x}+\lambda \mathbf{b}(s))=\exp \left(-\frac{\mathbf{b}(s)^{2}}{2 R}\right) \hat{g}(\mathbf{x}+\lambda \mathbf{b}(s))
$$

The proof of eq.(13) (for a Hamiltonian with a potential) was given in ref.[8]. The operator (11) coincides with the one for the Schrödinger equation with the potential $\frac{1}{2} P g P$.

Eq.(13) can be considered as an analytic continuation of the imaginary time version (the diffusion equation [10])

$$
-\partial_{\tau} \psi_{\tau}(\mathbf{x}, P)=\tilde{\mathcal{A}} \psi_{\tau}(\mathbf{x}, P)
$$

However, with $\hat{g}^{00}=-1+\hat{h}^{00}$ we would need to restrict ourselves to $\hat{h}^{00}$ which are bounded from below if the diffusion equation (16) is to make sense. This is the basic reason for an analytic continuation from the Wiener to the Feynman integral in the final formulas in this paper. In contradistinction to the standard 
methods we achieve the analytic continuation in time through an analytic continuation in space. We would not need to require any analycity of the potential if we worked with the imaginary time. We would obtain the formulas (13) and (14) with $\lambda=1$. Then, an analytic continuation to complex $\lambda=\frac{1}{\sqrt{2}}(1+i)$ is needed. Both the imaginary time and the real time formulas follow from the Trotter product formula. Denoting

$$
\mathcal{A}=-\frac{1}{2} \triangle+V
$$

we have for the imaginary time

$$
\exp (-\tau \mathcal{A})=\lim _{n \rightarrow \infty}\left(\exp \left(\frac{\tau}{2 n} \triangle\right) \exp \left(-\frac{\tau}{n} V\right)\right)^{n}
$$

The kernel $K$ of the r.h.s. of the formula (18) can be expressed by the Brownian motion. In the real time case we express the kernel (for a finite $n$ ) by means of the free propagators and rotate the integration axis from $x$ to $\lambda x$. Then, the limit $n \rightarrow \infty$ can again be expressed by the Brownian motion (as in eq.(13)). We can express the solution of eq.(13) by means of the Feynman kernel $K$ defined by $\psi_{\tau}(x)=\int d y K_{\tau}(x, y) \psi(y)$. We have [8][9][13]

$$
\begin{aligned}
& K_{\tau}(x, y)=(2 \pi)^{-D+d}(2 \pi i \tau)^{-\frac{d}{2}} \exp \left(\frac{i}{2 \tau}(\mathbf{y}-\mathbf{x})^{2}\right) \int d P \exp (i P(Y-X)) \\
& \exp \left(-i \frac{\tau}{2} P^{2}\right) E\left[\exp \left(-\frac{i}{2} \int_{0}^{\tau} P_{\mu} \hat{h}^{\mu \nu}(\mathbf{v}(s, \mathbf{x}, \mathbf{y})) P_{\nu} d s\right)\right]
\end{aligned}
$$

where

$$
\mathbf{v}(s, \mathbf{x}, \mathbf{y})=\mathbf{x}+\frac{s}{\tau}(\mathbf{y}-\mathbf{x})+\lambda \sqrt{\tau} \mathbf{a}\left(\frac{s}{\tau}\right)
$$

and $\mathbf{a}$ is the Brownian bridge starting from 0 and ending at $s=\tau$ in 0 . The Brownian bridge is defined as the Gaussian process with mean equal to zero and the covariance

$$
E\left[a_{j}(s) a_{k}\left(s^{\prime}\right)\right]=\delta_{j k} s\left(1-s^{\prime}\right)
$$

for $s \leq s^{\prime}$. The representation [13] $\mathbf{a}(s)=(1-s) \mathbf{b}\left(\frac{s}{1-s}\right)$ (where $\mathbf{b}$ is the Brownian motion) is useful for computations.

\section{An average over the quantum metric}

We are interested in metrics which are relativistic quantum fields. In the realtime quantum field theory the time-ordered products have the Källen-Lehmann representation [14]

$$
\begin{aligned}
& \mathcal{D}^{\mu \nu ; \alpha \beta}(x, y)=\left\langle 0\left|T\left(h^{\mu \nu}(x) h^{\alpha \beta}(y)\right)\right| 0\right\rangle=\int d \rho\left(m^{2}\right) \mathcal{D}_{F}^{\mu \nu ; \alpha \beta}\left(x-y ; m^{2}\right) \\
& =\lim _{\epsilon \rightarrow 0} \mathcal{P}^{\mu \nu ; \alpha \beta} \int d \rho\left(m^{2}\right) \int_{0}^{\infty} i d \tau(2 \pi i \tau)^{-\frac{d}{2}} \exp \left(-\frac{i}{2} m^{2} \tau-\epsilon \tau\right) \exp \left(\frac{i}{2 \tau}(x-y)^{2}\right)
\end{aligned}
$$


Hence,

$$
\begin{aligned}
& \mathcal{D}_{F}(x-y)=\int d \rho\left(m^{2}\right) \mathcal{D}_{F}\left(x-y ; m^{2}\right) \\
& =\lim _{\epsilon \rightarrow 0} \int_{0}^{\infty} i d \tau \sigma(\tau) \exp (-\epsilon \tau) \exp \left(\frac{i}{2 \tau}(x-y)^{2}\right)
\end{aligned}
$$

where

$$
\sigma(\tau)=\int d \rho\left(m^{2}\right)(2 i \pi \tau)^{-\frac{d}{2}} \exp \left(-\frac{i}{2} m^{2} \tau\right)
$$

The Euclidean version of eq.(23) reads

$$
\mathcal{D}(\mathbf{x}-\mathbf{y})=\int_{0}^{\infty} d \tau \sigma(-i \tau) \exp \left(-\frac{1}{2 \tau}(\mathbf{x}-\mathbf{y})^{2}\right)
$$

It can be seen that if

$$
\mathbf{x}-\mathbf{y} \rightarrow \mathbf{x}-\mathbf{y}+\mathbf{z}
$$

then we can continue to complex $\mathbf{z}$ if

$$
\Re\left((\mathbf{x}-\mathbf{y}+\mathbf{z})^{2}\right) \geq 0
$$

We can formulate the analycity also in terms of the radial analycity meaning that $\mathbf{x}-\mathbf{y} \rightarrow \lambda(\mathbf{x}-\mathbf{y})$ if $\Im\left(\lambda^{2}(\mathbf{x}-\mathbf{y})^{2}\right) \geq 0$. From the point of view of the propertime representation in the imaginary time formulation it is useful to introduce the regularized imaginary time metric fields in the form

$$
h_{\delta}(\mathbf{x})=\int_{\delta}^{\infty} d \tau \exp \left(-\frac{\tau}{4} \mathbf{k}^{2}\right) \chi(\mathbf{k}, \tau) \sqrt{\sigma(-i \tau)} \exp (i \mathbf{k x})
$$

where

$$
\left\langle\tilde{\chi}^{\mu \nu}(\mathbf{k}, \tau) \tilde{\chi}^{\rho \sigma}\left(\mathbf{k}^{\prime}, \tau^{\prime}\right)\right\rangle=\mathcal{P}^{\mu \nu ; \rho \sigma} \delta\left(\mathbf{k}+\mathbf{k}^{\prime}\right) \delta\left(\tau-\tau^{\prime}\right)
$$

Then, $h_{\delta}(\mathbf{x})$ is an analytic function of $\mathbf{x}$ and its correlation function is $\mathcal{D}_{\delta}$ where in eq. (25) the $\tau$-integral starts from $\tau \geq \delta$. For further computations we assume that the metric is Gaussian. We have seen in eq.(8) that an average over the scalar field leads to a determinant of $\mathcal{A}$ which depends on the metric $g$. It is difficult to calculate non-perturbatively a contribution of the non-Gaussian terms in the complete theory of the interaction of gravity with a scalar field. We are able to calculate the averages over the metric only through an expansion in the number of closed scalar loops as discussed at the end of sec.2. In such an expansion the ultraviolet behaviour does not depend on the number of loops. Hence, we may restrict ourselves here to the zeroth order corresponding to $\operatorname{det} \mathcal{A}=1$.

Using the Trotter product formula (18) (with $V=\frac{1}{2} P g P$ and $\tau \rightarrow-i \tau$ ) we obtain (for a regular Gaussian metric (26), see eq.(8) for a calculation of the Gaussian average)

$$
\begin{aligned}
& \left\langle 0\left|\left(\exp \left(\frac{i \tau}{2 n} \triangle\right) \exp \left(-\frac{i \tau}{n} V\right)\right)^{n}\right| 0\right\rangle_{g r}(X, \mathbf{x} ; Y, \mathbf{y}) \\
& =\int d P \exp (i P(X-Y))\left(2 \pi i \frac{\tau}{n}\right)^{-\frac{d}{2}} \exp \left(i n \frac{\left(\mathbf{x}-\mathbf{x}_{1}\right)^{2}}{2 \tau}\right) \\
& \ldots . \exp \left(-\frac{\tau^{2}}{8 n^{2}} \sum P P \mathcal{D}_{\delta}\left(\mathbf{x}_{j}-\mathbf{x}_{j-1}\right) P P\right) d \mathbf{x}_{1} \ldots d \mathbf{x}_{n}
\end{aligned}
$$


In this equation we rotate the integration line $\mathbf{x} \rightarrow \lambda \mathbf{x}$. Then, the limit $n \rightarrow \infty$ can be expressed by the Brownian motion. As applied to propagators instead of the kernel $\mathcal{A}^{-1}(x, y)$ consider $\mathcal{A}^{-1}(\lambda \mathbf{x}, X ; \lambda \mathbf{y}, Y)$. Using the Brownian bridge representation we obtain for an average (28) over the metric (22)

$$
\begin{aligned}
& \left\langle\mathcal{A}^{-1}(\lambda \mathbf{x}, X ; \lambda \mathbf{y}, Y)\right\rangle=\int_{0}^{\infty} d \tau \exp (i P(X-Y))(2 \pi \tau)^{-\frac{d}{2}} \exp \left(-\frac{1}{2 \tau}(\mathbf{x}-\mathbf{y})^{2}\right) \\
& E\left[\exp \left(-\frac{1}{8} \int_{0}^{\tau} \int_{0}^{\tau} d s d s^{\prime} P P \mathcal{D}_{\delta}\left(\lambda\left(\tilde{\mathbf{v}}(s)-\tilde{\mathbf{v}}\left(s^{\prime}\right)\right) P P\right)\right]\right.
\end{aligned}
$$

where

$$
\tilde{\mathbf{v}}(s)=\mathbf{x}+\frac{s}{\tau}(\mathbf{y}-\mathbf{x})+\sqrt{\tau} \mathbf{a}\left(\frac{s}{\tau}\right)
$$

We can calculate higher order correlations

$$
\begin{aligned}
& \left\langle\mathcal{A}^{-1}(\lambda \mathbf{x}, X ; \lambda \mathbf{y}, Y) \mathcal{A}^{-1}\left(\lambda \mathbf{x}^{\prime}, X^{\prime} ; \lambda \mathbf{y}^{\prime}, Y^{\prime}\right)\right\rangle=\int_{0}^{\infty} d \tau_{1} \int_{0}^{\infty} d \tau_{2} d P d P^{\prime} \\
& \exp (i P(X-Y))\left(2 \pi \tau_{1}\right)^{-\frac{d}{2}} \exp \left(-\frac{1}{2 \tau_{1}}(\mathbf{x}-\mathbf{y})^{2}\right) \exp \left(i P^{\prime}\left(X^{\prime}-Y^{\prime}\right)\right)\left(2 \pi \tau_{2}\right)^{-\frac{d}{2}} \\
& \exp \left(-\frac{1}{2 \tau_{2}}\left(\mathbf{x}^{\prime}-\mathbf{y}^{\prime}\right)^{2}\right) \\
& E\left[\operatorname { e x p } \left(-\frac{1}{8} \int_{0}^{\tau_{1}} \int_{0}^{\tau_{1}} d s d s^{\prime} P P \mathcal{D}_{\delta}\left(\lambda\left(\tilde{\mathbf{v}}(s)-\tilde{\mathbf{v}}\left(s^{\prime}\right)\right) P P\right.\right.\right. \\
& -\frac{1}{8} \int_{0}^{\tau_{2}} \int_{0}^{\tau_{2}} d s d s^{\prime} P^{\prime} P^{\prime} \mathcal{D}_{\delta}\left(\lambda\left(\tilde{\mathbf{v}}^{\prime}(s)-\tilde{\mathbf{v}}^{\prime}\left(s^{\prime}\right)\right) P^{\prime} P^{\prime}\right. \\
& \left.+\frac{1}{4} \int_{0}^{\tau_{2}} \int_{0}^{\tau_{1}} d s d s^{\prime} P P \mathcal{D}_{\delta}\left(\lambda\left(\tilde{\mathbf{v}}^{\prime}(s)-\tilde{\mathbf{v}}\left(s^{\prime}\right)\right) P^{\prime} P^{\prime}\right)\right] \\
& +\left(x \rightarrow x^{\prime}\right)
\end{aligned}
$$

Here, $\left(x \rightarrow x^{\prime}\right)$ means the same expression with $x$ exchanged with $x^{\prime}, \tilde{\mathbf{v}}^{\prime}$ is an independent bridge with $(\mathbf{x}, \mathbf{y}) \rightarrow\left(\mathbf{x}^{\prime}, \mathbf{y}^{\prime}\right)$. In ref.[15] we have shown that the formulas (29)-(30) come from a resummation of the perturbation series in $\hat{h}$ of $\square_{g}^{-1}$. It follows from eq.(8) that this is also a resummation of the Dyson series in the interaction Hamiltonian. We could derive the short distance behaviour of the 4-point function from eq.(30). It can be shown [5] that its singularity is a product of the singularities of the two-point functions.

Whether the correlations (29)-(30) are finite or not depends on whether the argument in the exponent is a well-defined function and whether the exponential factors are integrable. The functions in the exponent are analytic continuations from real arguments. It will be useful to use a concrete representation of these functions. So, for our special case of the $|\mathbf{x}-\mathbf{y}|^{-1}$ metric correlations we consider the representation

$$
\left(\frac{1}{|\mathbf{k}|}\right)^{2}=\int_{0}^{\infty} d r \exp \left(-\frac{1}{2} r|\mathbf{k}|^{2}\right)\left(\int_{0}^{\infty} d r \exp \left(-\frac{1}{2} r\right)\right)^{-1}
$$

Then, we can perform the integral over $\mathbf{k}$ (with the regularization (26))

$$
\begin{aligned}
& \mathcal{D}_{\delta}(\mathbf{x}-\mathbf{y})=\int_{\delta}^{\infty} d r \int d \mathbf{k} \exp \left(-\frac{1}{2} r|\mathbf{k}|^{2}\right) \exp (i \mathbf{k}(\mathbf{x}-\mathbf{y}))= \\
& \int_{\delta}^{\infty} d r(2 \pi r)^{-\frac{d}{2}} \exp \left(-\frac{1}{2} r^{-1}(\mathbf{x}-\mathbf{y})^{2}\right)
\end{aligned}
$$


From the representation (32) it can be seen that this is an analytic function of $\mathbf{z}^{2}=(\mathbf{x}-\mathbf{y})^{2}$ as long as $\Re \mathbf{z}^{2} \geq 0$ (the equality sign is allowed only if $\Im \mathbf{z}^{2} \neq 0$ ). The argument of the exponential factors in eqs.(29)-(30) is a well-defined random variable because it is square integrable. In fact, the expectation value

$$
\begin{aligned}
& E\left[\mid \int d s d s^{\prime} \mathcal{D}_{\delta}\left(\left.\lambda\left(\tilde{\mathbf{v}}(s)-\tilde{\mathbf{v}}\left(s^{\prime}\right)\right)\right|^{2}\right]\right. \\
& =\int d s d s^{\prime} d t d t^{\prime} E\left[\overline{\mathcal{D}_{\delta}\left(\lambda\left(\tilde{\mathbf{v}}(s)-\tilde{\mathbf{v}}\left(s^{\prime}\right)\right)\right.} \mathcal{D}_{\delta}\left(\lambda\left(\tilde{\mathbf{v}}(t)-\tilde{\mathbf{v}}\left(t^{\prime}\right)\right)\right]\right.
\end{aligned}
$$

can be explicitly calculated (using eq.(21)) and shown to be finite for $\delta>0$ as well as in the limit $\delta \rightarrow 0$. When $\delta=0$ the complex argument in the exponent in eqs.(29)-(30)) for the propagator (32) scales as

$$
-\mathcal{D}\left(\lambda\left(\tilde{\mathbf{v}}(s)-\tilde{\mathbf{v}}\left(s^{\prime}\right)\right)\right)=-\frac{1}{\lambda} \mathcal{D}\left(\tilde{\mathbf{v}}(s)-\tilde{\mathbf{v}}\left(s^{\prime}\right)\right)
$$

where $\mathcal{D}$ is positive. Hence, the real part of (34) is negative supplying a damping factor for the integrals of the exponential factors (29)-(30).

After the proof that the expressions (29)-(30) are finite we obtain just by scaling ( as in [5], although $g^{\mu \nu}=\eta^{\mu \nu}+h^{\mu \nu}$ is not scale invariant)

$$
\left\langle\mathcal{A}^{-1}(X, Y)\right\rangle \simeq|X-Y|^{-2-\frac{1}{3}}
$$

at short distances (and in the approximation $\operatorname{det} \mathcal{A}=1$ ).

For the behaviour in the $\mathbf{x}$ direction we put $X=Y=0$ then

$$
\left\langle\mathcal{A}^{-1}(\mathbf{x}, \mathbf{y})\right\rangle=R|\mathbf{x}-\mathbf{y}|^{-2+\frac{1}{4}}
$$

where $R$ is a constant. It is more regular than the two-point function for the four-dimensional free field (see ref.[16] for a more general argument). In order to treat the general case of $2 n$-point functions and $\operatorname{det} \mathcal{A} \neq 1$ we expand $\operatorname{det} \mathcal{A}$ in eq.(8) in terms of the Green's functions as discussed at the end of sec.2. Then, at each order we can calculate the expectation values of products of Green's functions (as in eq.(30), see also [5]). In each order of the expansion of $\operatorname{det} \mathcal{A}$ the results (35)-(36) can be generalized to arbitrary $2 n$-point correlation functions proving their anomalous short distance behaviour.

\section{Discussion}

We have discussed a Hamiltonian formulation of an interaction of a quantum metric with a quantum scalar field. We have compared the standard Dyson expansion for this model with the results coming from the functional integral. We have calculated scalar field correlation functions in the Gaussian approximation for the metric field expanding the correlation functions in the number of closed scalar loops. The ultraviolet behaviour does not depend on the number of loops. We have shown that at each order of the expansion the short distance 
behaviour is substantially modified as a result of the interaction with gravitons. In particular, the behaviour at equal times of the correlation functions is more regular than the one for the free scalar field. In sec. 4 we concentrated on the static metric corresponding to $D=d+1=4$ of the framework of refs.[4]-[6] because it may be relevant to physical models of gravitons in equilibrium with matter. The Hamiltonian framework of this paper may be considered for any $d<D$. From the S-matrix formula we can obtain (inserting static sources in eq. (8); see also a more detailed discussion in [14] ) the expression for the Yukawa potential

$$
V(\mathbf{x}, \tilde{\mathbf{X}})=\int_{-\infty}^{\infty} d X_{D}\left\langle\mathcal{A}^{-1}\left(\mathbf{x}, \tilde{\mathbf{X}}, X_{D}\right)\right\rangle
$$

here we denoted $X=\left(\tilde{\mathbf{X}}, X_{D}\right)$, where $X_{D}$ is the time. If $\mathcal{D}(\mathbf{x}) \simeq|\mathbf{x}|^{-4 \gamma}$ at short distances then using just scaling properties of the formula (29) (as in refs.[4]-[6]) we can conclude that

$$
V(\mathbf{x}, \tilde{\mathbf{X}}=0) \simeq|\mathbf{x}|^{2-d-(1-\gamma)(D-d-1)}
$$

Hence, in general the Yukawa potential is less singular in the $\mathbf{x}$ direction than the canonical one ( except the static case of sec. 4 corresponding to $D=d+1$ when the Yukawa potential does not change in the $\mathbf{x}$ direction at short distances).

In the $\tilde{\mathbf{X}}$ direction (if $D>d+1$ )

$$
V(\mathbf{x}=0, \tilde{\mathbf{X}})=|\tilde{\mathbf{X}}|^{-D+\frac{3}{1-\gamma}-(d+1) \frac{\gamma}{1-\gamma}}
$$

The distance scale at which the anomalous behaviour would appear is determined by the scaling behaviour of the metric field. In Einstein gravity there is a dimensional parameter of the Planck scale. In this case the eventual change of the short distance behaviour is expected below this length scale.

\section{References}

[1] S. Deser, Rev.Mod.Phys.29,417(1957)

[2] C.J. Isham,Abdus Salam and J. Strathdee, Phys.Rev.D3,1805(1971)

[3] L.H.M. Ford,Phys.Rev. D51,1692(1995)

[4] Z. Brzezniak and Z. Haba, Journ.Phys.A34,L139(2001)

[5] Z. Haba, Journ.Phys.A35,7425(2002)

[6] Z. Haba, Journ.Math.Phys.43,5483(2002)

[7] S. Weinberg, Phys.Rev.138,B988(1965)

[8] Z. Haba, Journ.Phys. A27,4247(1994) 
[9] Z. Haba, The Feynman Integral and Random Dynamics in Quantum Physics, Kluwer, Dordrecht, 1999

[10] N. Ikeda and S. Watanabe, Stochastic Differential Equations and Diffusion Processes, North Holland,1981

[11] S.D. Majumdar, Phys.Rev.73,390(1947)

[12] D. Kramer, H. Stephani, M. MacCallum and E. Herlt, Exact Solutions of the Einstein Field Equations, Deutsch.Verlag Wiss.,Berlin,1980

[13] B. Simon, Functional Integration and Quantum Physics, Academic Press, 1979

[14] I. Bialynicki-Birula and Z. Bialynicka-Birula, Quantum Electrodynamics, Pergamon Press, Oxford, 1975

[15] Z. Haba, hep-th/0306015

[16] Z. Haba,Phys.Lett.B528,129(2002) 